\title{
Innocampus Explora: Nuevas formas de comunicar ciencia
}

José Moros Gregorio ${ }^{\text {e1 }}$, Paula Rodrigo Martínez ${ }^{\text {e1 }}$, Cristina Torres Piedras ${ }^{\text {e2 }}$, Lucía Montoya Martínez ${ }^{\text {e2 }}$, Jorge Peña Peña ${ }^{\text {e3 }}$, Marta Pla Díaz ${ }^{\text {e3 }}$, Paula Galarza Jiménez ${ }^{\text {e3 }}$, Dalal-Qamar Bahra Al-Borghli ${ }^{\mathrm{e}}$, Virgínia Yeves Márquez ${ }^{\mathrm{e}}$, María Ibañez Jaime ${ }^{\mathrm{e}}$, Borja Castillo Caballero ${ }^{\text {e6 }}$, Dídac Cuenca Peris ${ }^{\text {e6 }}$, Ana Chirivella Juan ${ }^{\text {e6 }}$, Elisa Pardo Cuenca ${ }^{\text {e6}}$, M. Luisa Cervera Sanz ${ }^{\text {p1 }}$, Rafael Ibáñez Puchades ${ }^{\text {p1 }}$, Rafael García Gil ${ }^{\text {p2 }}$, Teresa Garrigues Pelufo ${ }^{\mathrm{p} 4}$, Javier Pereda Cervera ${ }^{\mathrm{p} 4}$, Nuria Garro Martínez ${ }^{\mathrm{p} 5}$, Santiago Garcia Lazaro $^{\mathrm{p} 5}$, Ana Corberán Vallet ${ }^{\mathrm{p} 6}$, Juanjo Peiró Ramada ${ }^{\mathrm{p} 6}$, Inmaculada García Robles ${ }^{\text {pc3 }}$, Luís F. Pascual Calaforra ${ }^{\text {pc3 }}$. ${ }^{\text {EEstudiante. }}$ PProfesor. ${ }^{\text {pc} P r o f e s o r ~ C o o r d i n a d o r . ~}$ ${ }^{1}$ Facultad de Química. ${ }^{2}$ Escola Tècnica Superior d'Enginyeria (ETSE-UV). ${ }^{3}$ Facultad de Ciencias Biológicas. ${ }^{4}$ Facultad de Farmacia. ${ }^{5}$ Facultad de Física. ${ }^{6}$ Facultad de Matemáticas.

Correo institucional del proyecto: innocampus@uv.es.

\begin{abstract}
Innocampus Explora aims to show the students of the Burjassot-Paterna campus of the Universitat de València how the different scientific degrees are interrelated. To do this we propose activities in which students and teachers work together to cover the interdisciplinary nature of science, both in everyday and professional issues. Throughout this course the activities developed relate to new ways to communicate science. With the development of this project we contribute to a transversal quality education for all the participating students.
\end{abstract}

Keywords: interdisciplinariety, science, divulgation, interactivity, collaboration, cinema

\section{Resumen \\ Innocampus Explora tiene por objetivo mostrar a los estudiantes del campus de Burjassot-Paterna de la Universitat de València cómo los diferentes grados científicos están interrelacionados. Para ello proponemos actividades en las que estudiantes y profesores trabajen conjuntamente para abarcar la interdisciplinariedad de la ciencia, tanto en temas cotidianos como profesionales. A lo largo de este curso las actividades desarrolladas se relacionan con las nuevas formas de comunicar ciencia. Con el desarrollo de este proyecto contribuimos a una formación transversal de calidad para todos los estudiantes participantes.}

Palabras clave: Innovación, interdisciplinariedad, ciencia, divulgación, interactividad, colaboración, cine. 


\section{Introducción}

La interdisciplinariedad, presente tanto en la actividad académica como científica desarrollada en el campus de Burjassot-Paterna, se pone continuamente de manifiesto con un buen número de ejemplos como son los ciclos de conferencias programados en los grados, las actividades formativas propuestas por diferentes instituciones o colectivos, las presentaciones de tesis doctorales o los propios proyectos de investigación desarrollados en los centros del campus. Ante la evidente relación entre el avance del conocimiento y la necesidad de combinar conocimientos y habilidades cada vez más específicas (Carvajal, 2010; Llano, 2016) pensamos que, la implementación de mecanismos que promuevan de manera activa la relación entre los estudiantes de las diversas titulaciones del campus de Burjassot-Paterna puede contribuir a fomentar esta cultura de colaboración tan necesaria para el desarrollo profesional de nuestros graduados en la sociedad actual. Nació así Innocampus Explora Burjassot-Paterna, un proyecto-plataforma de unión y coordinación entre los centros del campus de Ciencias que tiene como objetivo fomentar actividades divulgativas de carácter científico donde se manifiesta la necesaria interdisciplinariedad. Este proyecto pretende transmitir a los estudiantes de todos los grados de ciencias e ingeniería de la Universitat de València la cultura de la interdisciplinariedad como un elemento y habilidad fundamental para poder innovar y transferir tecnología en la sociedad y economía globales de hoy en día. Pensamos que la posibilidad de transmitir estos valores y fomentar el contacto entre estudiantes de grado de las diferentes disciplinas científicas y tecnológicas de la universidad puede establecer el punto de partida de futuros emprendedores. Con el fin de articular este objetivo, también consideramos necesario implicar al Parque Científico de la Universitat de València, tanto sus institutos de investigación como sus empresas, en cuanto que incubadoras de startups.

El proyecto de innovación educativa denominado "Innocampus Explora" cuenta con financiación por parte del Servei de Formació Permanent i Innovació Educativa (SFPIE) de la Universitat de València además de una aportación por parte de todos los centros que participan. Tiene como objeto promover de manera activa la relación entre los estudiantes de las diversas titulaciones de ciencias e ingeniería de la Universitat de València, así como fomentar la cultura de colaboración en equipos interdisciplinares. La interdisciplinariedad permite una visión más completa de la ciencia, fomenta el intercambio de experiencias, la aportación de nuevas ideas y es imprescindible para el avance de la sociedad actual.

Las titulaciones universitarias implicadas son: grado en Física, grado en Óptica y Optometría, grado en Química, grado en Biología, grado en Bioquímica y Ciencias Biomédicas, grado en Biotecnología, grado en Ciencias Ambientales, grado en Matemáticas, grado en Ciencia y Tecnología de los Alimentos, grado en Ciencias Gastronómicas, grado en Farmacia, grado en Nutrición Humana y Dietética, doble grado en Farmacia y en Nutrición Humana y Dietética, grado en Ingeniería Electrónica Industrial, grado en Ingeniería Electrónica de Telecomunicación, grado en Ingeniería Informática, grado en Ingeniería Multimedia, grado en Ingeniería Química, grado en Ingeniería Telemática y grado en Ciencia de Datos. 
José Moros Gregorio, Paula Rodrigo Martínez, Cristina Torres Piedras, Lucía Montoya Martínez, Jorge Peña Peña, Marta Pla Díaz, Paula Galarza Jiménez, Dalal-Qamar Bahra Al-Borghli,

Virgínia Yeves Márquez, María Ibañez Jaime, Borja Castillo Caballero, Dídac Cuenca Peris, Ana Chirivella Juan, Elisa Pardo Cuenca, M. Luisa Cervera Sanz, Rafael Ibáñez Puchades, Rafael García Gil, Teresa Garrigues Pelufo, Javier Pereda Cervera, Nuria Garro Martínez,

Santiago Garcia Lazaro, Ana Corberán Vallet, Juanjo Peiró Ramada, Inmaculada García Robles,

\section{Objetivos}

Luís F. Pascual Calaforra

Innocampus Explora presenta los siguientes objetivos:

- Demostrar a estudiantes de grados de ciencias la interrelación existente entre los mismos y la interdisciplinariedad profesional que se puede desarrollar en base a sus estudios.

- Mostrar la presencia de la ciencia en el día a día de las personas, planteando una visión analítica y científica en temas cotidianos.

- Divulgar conocimientos científicos concretos, desde una visión multidisciplinar, simplificándolos para hacerlos comprensibles a estudiantes y profesionales de diversas ramas de la ciencia.

- Fomentar el trabajo y gestión de un equipo interdisciplinar, formado por estudiantes y profesores, rompiendo la barrera del rol establecido por el entorno educativo.

- Mostrar a los estudiantes que los conocimientos impartidos en grados diferentes al que cursan pueden resultar de gran utilidad en su desarrollo profesional.

- Servir de plataforma para todas aquellas iniciativas de los estudiantes encaminadas a fomentar la interdisciplinariedad.

\section{Desarrollo de la innovación}

\subsection{Innocampus Explora en los cursos anteriores}

Fue durante el curso 2015-16 cuando la profesora Maria Luisa Cervera, primera coordinadora del proyecto, impulsó una comisión de trabajo con representantes del Personal Docente e Investigador de todos los centros del campus que permitió, tras la incorporación de una decena de estudiantes de los distintos centros, establecer un grupo de trabajo centrado en articular distintas actividades encaminadas a fomentar la interdiscplinariedad. Entre las primeras acciones organizativas desarrolladas, se crearon una página web institucional (https://www.uv.es/innocampus/es/innocampus.html, Fig. 1) y una cuenta de correo (innocampus@uv.es). Así mismo, los estudiantes crearon perfiles en Facebook y Twitter.
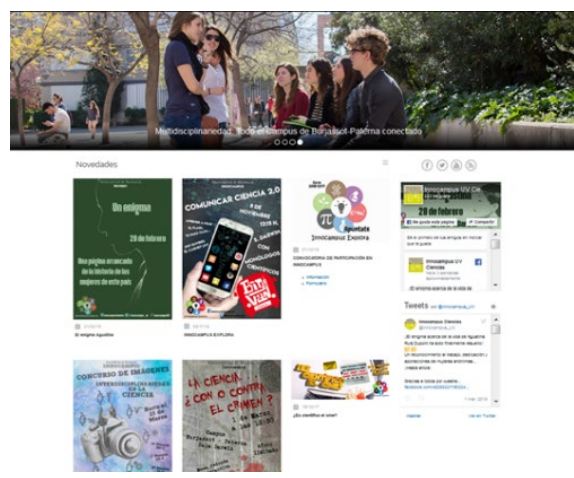

Fig. 1 Página web del proyecto Innocampus Explora. 
La primera actividad organizada (5 de mayo de 2016), encaminada tanto a presentar el proyecto como su objetivo central, el fomento de la interdisciplinariedad, se concretó en una mesa redonda sobre nanociencia y nanotecnología. Contó con la participación de profesores e investigadores de los diferentes centros e institutos de investigación del campus, quienes dieron una visión interdisciplinar y accesible de temas cercanos a su campo relacionados con el mundo de la nanotecnologia. Se consiguió dar difusión de la actividad en la página www.uv.es, en todas las páginas de los centros y en las pantallas de televisión del campus Burjassot-Paterna. Los estudiantes, además de ayudar a la dinamización y organización de la mesa redonda fueron los responsables del mantenimiento de las redes sociales (Facebook y Twitter) de Innocampus Explora.

Durante el curso 16-17 el proyecto siguió contando con la financiación del Vicerrectorado de Políticas de Formación y Calidad Educativa de la Universitat de València, gracias a la convocatoria de ayudas a planes de Innovación de Centro lo que permitió continuar con el desarrollo de nuestros objetivos, ahora bajo la coordinación del profesor Javier Pereda Cervera de la Facultad de Farmacia. Durante este curso, la Universitat acordó la concesión del reconocimiento de 1,5 créditos para los estudiantes integrados en el proyecto por su participación en la organización y difusión de las actividades tales como mesas redondas, conferencias, concursos.... Dicha solicitud fue avalada por las Comisiones Académicas de Titulo (CAT) de todas las titulaciones implicadas. El proyecto retomó su actividad en el campus de Burjassot-Paterna con la mesa redonda "La tierra más allá de la tierra" ( 20 de octubre de 2016) en la que estudiantes de los diferentes centros debatieron sobre los retos científico-tecnológicos que supondría la colonización de otro planeta. Analizaron el panorama científico actual en todo lo que implica un viaje espacial: relatividad, supervivencia durante periodos prolongados en el espacio, qué es posible encontrar, como emprender un viaje espacial... La segunda actividad de este curso fue la mesa redonda "Cerveza y ciencia" coordinada por los profesores (22 de marzo de 2017). En la misma se contó con la colaboración de empresas cerveceras artesanales valencianas, así como con la ayuda de los alumnos de Ciencias Gastronómicas. De esta forma, la mesa redonda fue acompañada de una cata de cervezas valencianas y un maridaje a cargo de los alumnos de Ciencias Gastronómicas que tuvo lugar en el hall de la Facultad de Farmacia y se organizó con tiques que previamente se habían repartido entre los asistentes a la mesa redonda (Fig. 2). Se consiguió visibilidad de esta actividad en la página www.uv.es, pantallas de televisión del Campus, redes sociales y páginas web de los diferentes centros, siendo además noticia en InfoUniversitat. Una tercera actividad durante este curso consistió en un concurso-certamen audiovisual donde los alumnos formaron grupos interdisciplinares de mínimo tres titulaciones a fin de realizar creaciones audiovisuales que expresen el trabajo en grupo interdisciplinar. Esta actividad llevó por título "Concurso de Imágenes- Interdisciplinariedad en la Ciencia". La entrega de premios fue el 5 de mayo de 2017 en el Salón de Grados de la Facultad de Matemáticas y las imágenes premiadas fueron expuestas de forma itinerante en el vestíbulo de los diferentes centros del campus y en Expociencia 2017, certamen abierto al público en general en el que científicos y emprendedores muestran experimentos, ideas y conocimientos de manera didáctica, interactiva y educativa. 
José Moros Gregorio, Paula Rodrigo Martínez, Cristina Torres Piedras, Lucía Montoya Martínez, Jorge Peña Peña, Marta Pla Díaz, Paula Galarza Jiménez, Dalal-Qamar Bahra Al-Borghli, Virgínia Yeves Márquez, María Ibañez Jaime, Borja Castillo Caballero, Dídac Cuenca Peris,

Ana Chirivella Juan, Elisa Pardo Cuenca, M. Luisa Cervera Sanz, Rafael Ibáñez Puchades, Rafael García Gil, Teresa Garrigues Pelufo, Javier Pereda Cervera, Nuria Garro Martínez,

Santiago Garcia Lazaro, Ana Corberán Vallet, Juanjo Peiró Ramada, Inmaculada García Robles, Luís F. Pascual Calaforra Durante el curso 17-18, una vez consolidado el proyecto-plataforma Innocampus Explora, se continuó con la realización de actividades que implican a todos los centros de BurjassotPaterna y fomentan la interdisciplinaridad y participación de estudiantes. Tras el éxito cosechado con las actividades del curso anterior, se decidió continuar con el proyecto dando rotación al coordinador responsable, asumiendo ahora este papel el profesor Juan Bautista Ejea de la Escuela Técnica Superior de Ingeniería, y manteniendo al menos un representante de cada centro. La Universitat volvió a conceder el reconocimiento de 1,5 créditos para los estudiantes integrados en el proyecto por su participación en la organización de las actividades. La primera en ser realizada consistió en la mesa redonda "¿Es científico el cine?" (26 de octubre de 2017), una actividad muy atractiva para amantes de la ciencia y del cine, que muy frecuentemente lo utilizan como recurso didáctico en el aula o la divulgación. Los ponentes fueron los propios estudiantes colaboradores del proyecto. Durante la misma, se proyectaron escenas de películas muy conocidas (aunque no siempre), al objeto de analizar, de forma interactiva, hasta qué punto el cine es científicamente riguroso. Los estudiantes de los centros del campus de Burjassot-Paterna pudieron debatir sobre los contenidos científicos presentados en las películas analizadas y a continuación se organizó una actividad interactiva, mediante la herramienta informática "Kahoot", de manera que los estudiantes participaron con sus móviles en una competición respondiendo a una serie de preguntas relacionadas con las escenas visualizadas. Resultó una actividad divertida en la que se pudo obtener conocimiento científico y también, cómo no, disfrutar del cine. Se entregó un obsequio a los 3 participantes que obtuvieron mayor puntuación (Fig. 3).

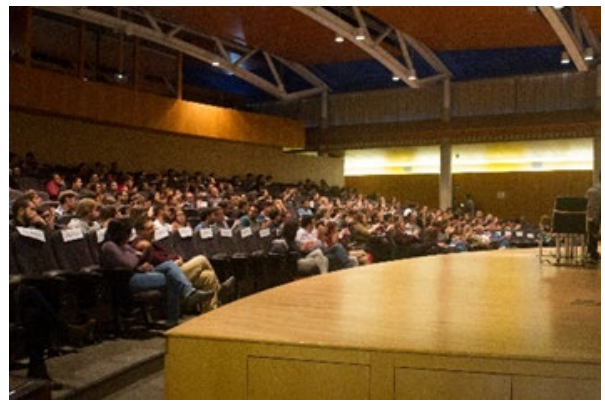

Fig. 2 Asistentes a la mesa redonda Cerveza y ciencia.

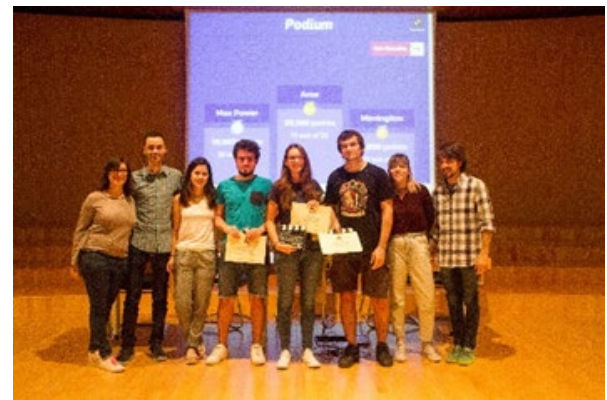

Fig. 3 ganadores del Kahoot de “ ¿Es científico el cine?" con alumnos de Innocampus Explora.

La segunda actividad fue una mesa redonda, coordinada por el profesorado, que tuvo por título "La ciencia... ¿con o contra el crimen?" (1 de marzo de 2018). Esta mesa contó con la intervención de profesores y profesionales que analizaron en qué medida la ciencia puede ayudar a cometer, pero también a resolver, actos delictivos. Se discutió si los conocimientos científicos que adquirimos en los diferentes grados científicos nos permiten acceder al ejercicio profesional contra el crimen en todas sus variantes. Nuevamente organizada por los alumnos miembros del proyecto, la mesa redonda contó con una competición interactiva mediante la herramienta informática "Kahoot". Se entregó un obsequio a los tres participantes que obtuvieron mayor puntuación. 


\subsection{El Proyecto en 2018-19}

Durante el presente curso, el Proyecto Innocampus Explora ha continuado su actividad focalizada en la visualización de la interdisciplinariedad en ciencia. Para ello se ha tomado como hilo conductor las "nuevas formas de comunicar ciencia" y se han organizado dos actividades complementarias. La primera de ellas, celebrada el 8 de noviembre (Fig. 4), ha consistido en la organización de la mesa redonda "Comunicar Ciencia" y en la misma los estudiantes han presentado y debatido sobre las distintas modalidades que actualmente se utilizan para comunicar ciencia: blogs, redes sociales, canales de youtube, literatura, cine, etc. En una segunda parte, se contó con la participación de los divulgadores de la "BigVan Ciencia" que presentaron dos monólogos científicos ampliamente celebrados (Fig. 5). La actividad fue seguida por cerca de 125 asistentes, la mayoría de los cuales la encontraron bastante $(58 \%)$ o muy interesante $(40 \%)$.

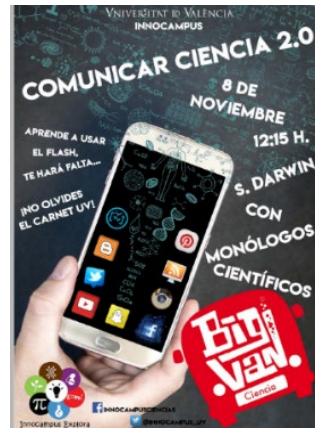

Fig. 4 Cartel promocional Mesa redonda "Comunicar ciencia".

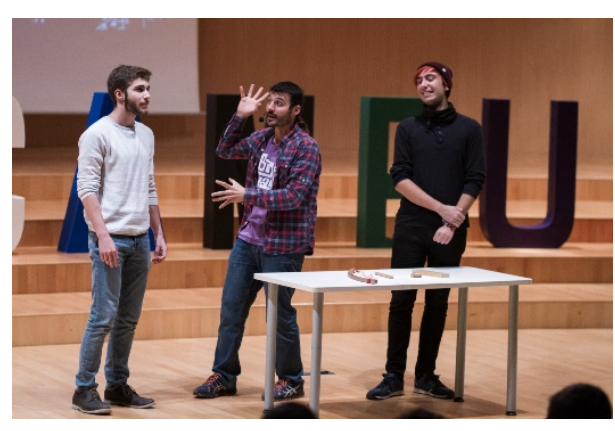

Fig. 5 Un momento de la actuación de BigVan Ciencia .

La segunda actividad, celebrada el 28 de febrero (Fig. 6), ha consistido en la proyección, y posterior coloquio, del documental "El enigma Agustina" dirigido por Manuel González y Emilio García (2018). El coloquio, apoyado por una presentación audiovisual preparada por la organización, se enfocó hacia las "otras Agustinas", las pioneras de la ciencia española que, en muchos casos fueron represaliadas tras la guerra civil y siempre poco reconocidas en su trabajo. Esta actividad nos ha permitido mostrar con un gran ejemplo, cómo el cine puede ser utilizado para comunicar ciencia siguiendo el camino recorrido por otros autores (Serrano, 2003; Gallego, 2007). Además, dada la temática y contenido de la película, también nos permitió tratar otros temas importantes como son el papel de la mujer en la ciencia española y la memoria histórica en relación con la actividad científica en nuestro país. La activdad fue seguida por algo más de 100 asistentes que además de criticar muy positivamente el documental la consideraron muy (77\%) o bastante interesante (14\%) (Fig. 7). 
José Moros Gregorio, Paula Rodrigo Martínez, Cristina Torres Piedras, Lucía Montoya Martínez, Jorge Peña Peña, Marta Pla Díaz, Paula Galarza Jiménez, Dalal-Qamar Bahra Al-Borghli, Virgínia Yeves Márquez, María Ibañez Jaime, Borja Castillo Caballero, Dídac Cuenca Peris, Ana Chirivella Juan, Elisa Pardo Cuenca, M. Luisa Cervera Sanz, Rafael Ibáñez Puchades, Rafael García Gil, Teresa Garrigues Pelufo, Javier Pereda Cervera, Nuria Garro Martínez, Santiago Garcia Lazaro, Ana Corberán Vallet, Juanjo Peiró Ramada, Inmaculada García Robles, Luís F. Pascual Calaforra

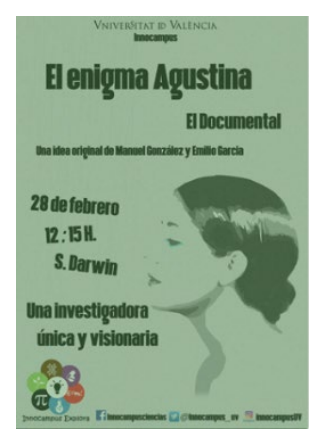

Fig. 6 Cartel promocional de la proyección del documental "El enigma Agustina.

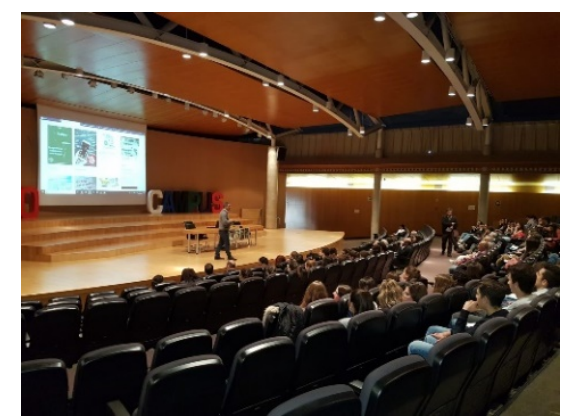

Fig. 7 Asistentes a la proyección del documental "El enigma Agustina".

\section{Análisis de los resultados}

En vista de lo anteriormente expuesto, el proyecto Innocampus Explora continua evolucionando desde un estado incipiente en el que tuvo poca visibilización hasta una fase de consolidación en los últimos cursos académicos. Gracias al incremento de los recursos económicos, que han pasado de los 750 euros en el primer curso (2015-16) a los 2600 en el actual, se ha podido realizar un mayor número de actividades $\mathrm{y}$, en consecuencia, dar una visibilidad continuada al proyecto.

Al objeto de analizar el interés que despiertan las actividades programadas entre los estudiantes, profesorado y personal de administración y servicios del campus y al mismo tiempo, para valorar hasta qué punto se alcanzan los objetivos propuestos, hemos realizado encuestas de satisfacción asociadas a cada una de las actividades. En primer lugar hemos observado, en lo que a asistencia se refiere, que se ha pasado de una asistencia reducida en la primera actividad programada (Nanociencia y nanotecnología) a un nivel de asistentes relativamente estable que podemos situar entre los cien o ciento cincuenta. Esta evolución, y el nivel de asistencia alcanzado, que deseamos mejorar, puede ser consecuencia en buena medida de la dificultad que tiene dar una buena difusión de las actividades así como conseguir motivar a los estudiantes para que participen en las mismas (figura 8).

Dada la impotancia de la difusión de las actividades hemos preguntado a los asistentes en relación a cómo han conocido las propuestas de Innocampus Explora en este curso y aunque pueda resultar ciertamente sorprendente, la gran mayoría se han enterado por canales de comunicación personales (el 30,4\% informado por un compañero, el $31 \%$ por un profesor) o tradicionales (el $15,2 \%$ por comunicaciones multimedia -carteles, videos-) y tan solo un $12 \%$ dice haberse enterado por páginas web y un 11,4\% por redes sociales (Facebook, Twitter o Instagram). 


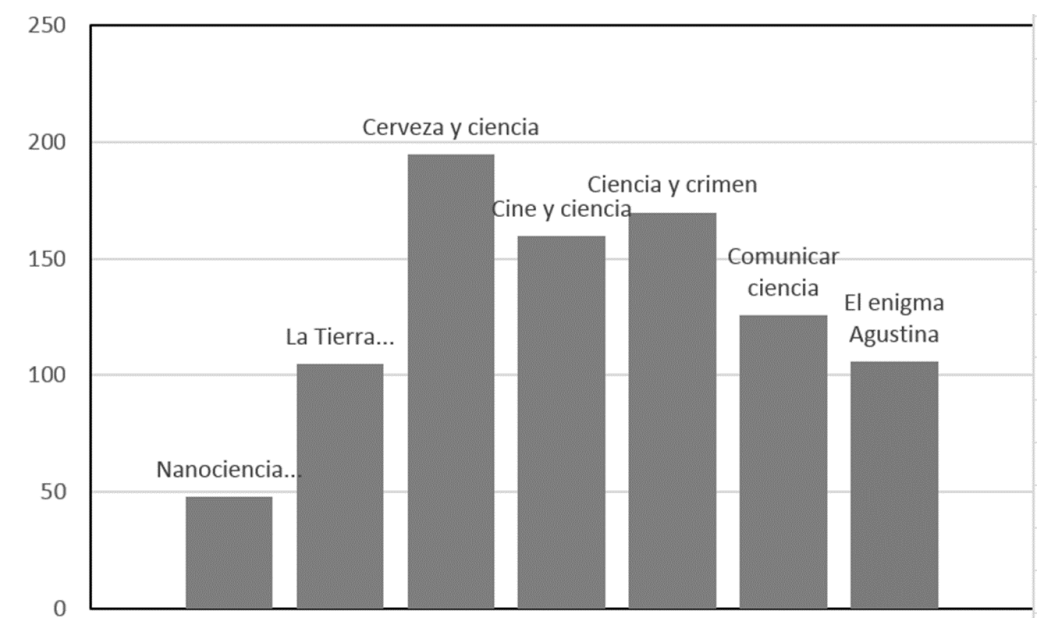

Fig. 8 Gráfica de asistencia a las diferentes actividades llevadas a cabo (en número de personas).

En lo relativo a la satisfacción con las actividades realizadas y para la evaluación de la formación y satisfacción del equipo Innocampus Explora, se ha preguntado a los asistentes su opinión sobre el grado de interés que les ha despertado la actividad, si les ha parecido adecuada a los objetivos del proyecto y si los contenidos y duración eran los adecuados. Respecto al grado de interés de las actividades relizadas este año, cerca del $80 \%$ de los asistentes lo han considerado alto o muy alto y el $16 \%$ lo han considerado medio. Tan solo el $4 \%$ de los asistentes no han visto satisfechas sus expectativas iniciales.

Por otra parte, en cuanto a la opinión manifestada por los asistentes respecto a si consideran las actividades adecuadas en relación con el objetivo del proyecto de fomentar la interdisciplinariedad y si les han satisfecho los contenidos y la duración de las mismas, la respuesta también ha sido muy positiva, con un $81,2 \%$ de encuestados que se manifiestan muy satisfechos con los contenidos propuestos como puede observarse en la figura 9.

Por último, también se ha preguntado a los asistentes si consideran Innocampus una plataforma efectiva para concienciar sobre la importancia de la interdisciplinariedad en la ciencia, mostrándose de acuerdo el $85,4 \%$, moderadamente de acuerdo el $13 \%$ y únicamente el $1,3 \%$ en desacuerdo, figura 9 .

\section{¿ES ADECUADA LA ORGANIZACIÓN ¿OBJETIVOS, CONTENIDOSY ¿ES INNOCAMPUSUNA DE LAS ACTIVIDADES ? DURACIÓN SON ADECUADOS? PLATAFORMA EFECTIVA?}
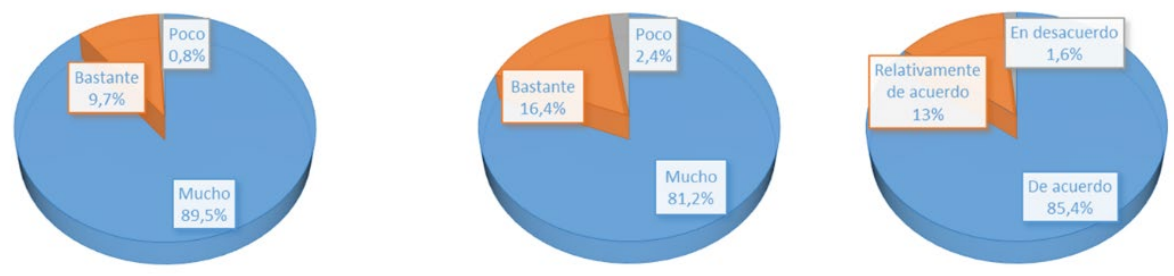

Fig. 9 Valoración de la adecuación de las actividades realizadas y de la idoneidad del proyecto para concienciar sobre la importancia de la interdisciplinariedad. 
José Moros Gregorio, Paula Rodrigo Martínez, Cristina Torres Piedras, Lucía Montoya Martínez, Jorge Peña Peña, Marta Pla Díaz, Paula Galarza Jiménez, Dalal-Qamar Bahra Al-Borghli, Virgínia Yeves Márquez, María Ibañez Jaime, Borja Castillo Caballero, Dídac Cuenca Peris, Ana Chirivella Juan, Elisa Pardo Cuenca, M. Luisa Cervera Sanz, Rafael Ibáñez Puchades, Rafael García Gil, Teresa Garrigues Pelufo, Javier Pereda Cervera, Nuria Garro Martínez,

Santiago Garcia Lazaro, Ana Corberán Vallet, Juanjo Peiró Ramada, Inmaculada García Robles, Luís F. Pascual Calaforra

\section{Conclusiones y propuestas de mejora}

En vista de lo anteriormente expuesto, el proyecto Innocampus Explora ha evolucionado desde un estado inicial relativamente modesto hasta su fase de consolidación en los últimos cursos académicos. Gracias al incremento de los recursos económicos destinados al fomento de este tipo de grupos, se ha podido realizar un mayor número de actividades, lo que ha permitido dar una mayor visibilidad al proyecto. En base a los resultados obtenidos podemos obtener algunas conclusiones interesantes, y plantear propuestas de mejora, relacionadas con tres aspectos fundamentales del proyecto como son $(i)$ el funcionamiento del grupo de trabajo, (ii) la respuesta por parte de estudiantes, profesores y personal de administración y servicios y (iii) el logro de los objetivos.

En cuanto al funcionamiento del grupo, tanto profesores como estudiantes valoramos el alto grado de colaboración entre los miembros del equipo a pesar de la dificultad que entraña poder organizar un colectivo bastante numeroso como es el de Innocampus. Así mismo, los estudiantes del equipo se muestran muy satisfechos por el trabajo realizado así como por el trabajo en competencias generales que han realizado para la organización de las actividades. Podemos estacar que la colaboración en el proyecto de miembros de todos los centros del campus posibilita una apreciable afluencia de público muy heterogéneo a las actividades lo que favorece el trabajo de la interdisciplinariedad. Consideramos que todavía hay mucho espacio para la mejora en el funcionamiento del grupo. Por tanto, el próximo curso queremos mejorar la estructura organizativa, tanto general como de los grupos de trabajo delegados para la organización de las distintas actividades. Con ello esperamos maximizar el rendimiento en la organización de las actividades y mejorar sensiblemente la obtención de información relacionada con el grado de satisfacción de los participantes en las actividades. En relación con la respuesta del público a las actividades programadas podemos concluir que ha sido muy positiva desde el punto de vista de los asistentes a las mismas. Pero por otra parte, también podemos constatar que la asistencia a las mismas podría haber sido mayor. Este hecho nos indica que, si bien cada año el proyecto es más conocido en el campus, debemos mejorar los canales de comunicación para publicitar las actividades -especialmente en lo referente a las redes sociales-, hacer un esfuerzo en programar actividades de interés y en concienciar de la conveniencia formativa de participar en este tipo de actividades extraacadémicas como parte importante de la formación universitaria. Es necesario, por tanto, compatibilizar los horarios de los distintos grados con la programación de actividades propuesta por Innocampus para facilitar la asistencia de los estudiantes. Así mismo consideramos importante mejorar la difusión a nivel de la propia Universitat y ampliarla al resto de universidades de nuestro entorno así como dedicar un esfuerzo especial para atraer a más profesorado, y especialmente al personal de administración y servicios, puesto que su participación es minoritaria.

Por último, y en relación con el grado de cumplimiento del objetivo principal el proyecto, visualizar la importancia de la interdisciplinariedad en la ciencia, consideramos que estamos contribuyendo significativamente a una formación transversal de calidad tanto de los 
estudiantes que participan de las actividades programadas como, y especialmente, de los estudiantes que a lo largo de los distintos cursos participan en el desarrollo del proyecto Innocampus Explora como miembros del equipo organizador.

Para los próximos cursos, está planeado que el proyecto continúe adelante con nuevos retos y desafíos, gracias a la ayuda económica de la Universitat de València y de los centros participantes.

\section{Referencias}

El enigma Agustina. (Dir. M. González y E. García). Instituto de Astrofísica de Andalucia, 2018. $<\mathrm{https} / / /$ www.iaa.csic.es/noticias/se-estrena-el-enigma-agustina-pelicula-producida-por-elinstituto-astrofisica-andalucia-iaa $>$ [Consulta 17 de enero de 2019]

CARVAJAL, Y. (2010). "Interdisciplinariedad: desafio para la educación superior y la investigación" en Luna Azul, n. 31 p. 156-169.

$<$ http://www.scielo.org.co/pdf/luaz/n31/n31a11.pdf $>$ [Consulta 21 de marzo de 2019]

GALLEGO, C. (2007). Tiem(pos)Modernos. Ensayos de Tecnociencia y cine. Madrid: Editorial Equipo Sirius, S. A.

LLANO, L., GUTIÉRREZ, M., STABLE, A., NÚÑEZ, M., MASÓ, M. y ROJAS, B. (2016). "La interdisciplinariedad: una necesidad contemporánea para favorecer el proceso de enseñanza aprendizaje" en Medisur, v.14 n. 3, p. 320-327.

$<$ http://www.medigraphic.com/cgi-bin/new/resumen.cgi?IDARTICULO=66798 $>$ [Consulta 14 de marzo de 2019]

SERRANO, J. M. (2003). De lo fantástico a lo real. Diccionario de la ciencia en el cine. España: Editorial Nivola.

Web del proyecto: www.uv.es/innocampus

Redes sociales: Twitter: @Innocampus_UV

Facebook:@innocampusciencias

(c) D EY-NC-ND 2019, Universitat Politècnica de València 